\title{
Revisión documental sobre casos significativos de comunicación en el campo de la salud y los hábitos de vida saludable en el ámbito académico ${ }^{1}$
}

\section{Literature review on significant cases of communication about health and healthy lifestyle habits in academic environments}

\section{Resúmen}

Este estudio se basó en la revisión documental de casos significativos a nivel nacional e internacional en los cuales se evidencia una articulación de la comunicación sobre el campo de la salud, específicamente con respecto a la línea aplicativa de los hábitos de vida saludable en un contexto académico. De 35 documentos revisados se seleccionaron 15 que respondieron a experiencias aplicadas en entornos universitarios. Inicialmente se abordan las experiencias internacionales, para luego centrarse en investigaciones en Colombia. La revisión evidenció unas metodologías de aplicación de la comunicación en los hábitos de vida saludable, lo cual devela la comunicación estratégica como herramienta clave para el cambio.

Palabras clave: comunicación y salud; hábitos de vida saludable; salud y entornos académicos; ámbitos académicos.

\section{Abstract}

This study was based on the review of national and international specific study cases on which an articulation of the health communication field is noticeable, focused on the application of healthy lifestyle habits in an academic context. Of 35 documents revised, 15 were selected that respond to experiences applied in university environments. Aspects of international experiences were analyzed in the first instance, and then research made in Colombia. The literature review evidenced methods for applying communication on healthy lifestyle habits, revealing strategic communication as a key tool for change.

Keywords: communication and health; healthy lifestyle habits; health and academic environments; academic fields.

\footnotetext{
1 El presente artículo surge como producto de la investigación denominada 'Los Hábitos de vida saludable en el entorno académico' adscrita al Programa de Comunicación Social-Periodismo de la Fundación Universitaria Católica Lumen Gentium.

2 José Fernando Ríos Espitia Comunicador Social-Periodista de la Universidad Autónoma de Occidente, con maestría en Salud Pública de la Universidad del Valle. Docente investigador tiempo completo del Programa de Comunicación Social-Periodismo de la Fundación Universitaria Católica Lumen Gentium. Correo electrónico: jrios@unicatolica.edu.co

3 Comunicador Social-Periodista de la Universidad Autónoma de Occidente. Magíster en Comunicación de la Universidad Autónoma de Occidente. Docente investigador tiempo completo del Programa de Comunicación Social-Periodismo de la Fundación Universitaria Católica Lumen Gentium. Correo electrónico: mavelasquez@unicatolica.edu.co
} 


\section{Introducción}

En este artículo se pretende dar cuenta de investigaciones desarrolladas en torno al concepto de la comunicación articulado con la salud y los hábitos de vida saludable, esencialmente en escenarios académicos universitarios; parte de contextos internacionales y posteriormente destaca estudios nacionales. Se tuvo en cuenta la relación que tienen las investigaciones mencionadas con aspectos que se puedan comprender desde el marco de los hábitos de vida saludable, tales como alimentación, ejercitación, consumo de sustancias, tabaquismo y alcohol, y el papel que jugó la comunicación en su indagación.

Inicialmente, se enuncian documentos que caracterizan experiencias investigativas en otros países y que además hacen énfasis -tanto en lo teórico, como en lo conceptual- en la relación entre la comunicación y el campo de la salud. Posteriormente, se detallan documentos que caracterizan experiencias de campañas que promueven hábitos de vida saludable, puntualmente en escenarios universitarios en Colombia. Finalmente, se describen investigaciones de pregrado y posgrado que relacionan la comunicación con el campo de la salud en la ciudad de Santiago de Cali.

\section{Metodología}

Para el desarrollo de esta indagación se revisaron documentos de las últimas dos décadas, con una recopilación y revisión inicial de más de 35 documentos; se seleccionaron entre diez y quince documentos para el presente estado del arte. Inicialmente, la revisión consistió en encontrar casos a nivel mundial, se le dio prelación a aquellos que tenían como objeto de estudio comunidades académicas universitarias, bien sea enfocadas en estudiantes o colaboradores. Posteriormente, se hizo una delimitación territorial para pasar de lo general a lo particular, a nivel continental y luego a nivel nacional. La selección de documentos se hizo acorde a la categorización de su contenido, se privilegiaron publicaciones con experiencias en comunidades académicas y que en sus conclusiones y resultados evidenciaban una articulación consistente entre la comunicación y el campo de la salud, que buscan experiencias puntuales sobre hábitos de vida saludable.

\section{Comunicación en salud: conceptualización y teoría}

Resulta interesante iniciar el recorrido de estudios y reflexiones entorno a la comunicación y la salud con el documento denominado "Comunicación en salud: conceptos y modelos teóricos" (Ríos Hernández, 2011), debido a que es una revisión teórica que logra analizar el concepto de la comunicación en el campo de la salud a partir de definiciones de organismos internacionales y expertos en el ámbito. El documento realiza un análisis bibliográfico y documental, recopila aspectos e investigaciones en torno al tema de comunicación y salud, con el propósito inicial de describir y posteriormente de adentrarse en un análisis crítico y reflexivo con respecto a las teorías que giran en torno al tema.

Inicialmente, el artículo afirma que para hablar de comunicación en salud resulta indispensable la compresión de las teorías que lo explican y que proponen maneras de cambio. En este sentido, como apertura aborda el comportamiento en tanto concepto y sus dos características fundamentales: 1) los comportamientos son influenciables con relación a diferentes factores; y 2) los comportamientos son de causalidad recíproca en cuanto a su entorno. Posteriormente, relaciona el comportamiento con el concepto de persuasión, señala que las teorías de la persuasión o del comportamiento evidencian un marco concreto para la comprensión del comportamiento humano.

El fin de ahondar en temas de comportamiento y persuasión corresponde a que el comportamiento humano permite comprender las razones por las cuales las personas desarrollan unas prácticas en 
torno al tema de la salud. Pero, además de eso, pueden ayudar a identificar factores externos que influyen en este y plantear procesos de cambio en dichas conductas. Uno de los aspectos que se resaltan en el texto es que muchos de los estudios que se desarrollan en torno al tema de la comunicación y la salud analizan al individuo en sí y dejan por fuera aspectos comunitarios relevantes y su papel como influenciadores externos del comportamiento. Es así que los estudios derivan en afirmaciones que hacen del individuo el elemento más importante para la generación de un cambio de conducta poblacional.

El autor postula además que debido al 'rol semiperiférico' que se le ha dado a la importancia de la comunicación en salud -al menos en la última década- se han presentado variaciones considerables en cuanto a lo teórico y lo metodológico. Aun así, en el artículo se vislumbra una clara intención de vincular la comunicación y la salud de manera teórica con la comunicación para el cambio social, al articular la temática con el poder que tiene la participación comunitaria como mecanismo para la prevención y el tratamiento de enfermedades. De este modo, se enlaza el campo de la comunicación para el cambio con el concepto de conducta para poder teorizar lo que corresponde con la comunicación y la salud. Reconoce así en el campo de la comunicación para el cambio un modelo que relaciona la comunicación con el desarrollo de las comunidades.

Para lo anterior, Ríos Hernández (2011), desarrolla una recopilación teórica sobre el campo de la comunicación para el cambio social, trae a colación a autores como Barranquero y Sáenz, quienes consideran que este tipo de comunicación sirvió para aunar diversos puntos de vista y voluntades de actores sociales; y a Gumucio Dagrón que establece la base sobre la comunicación para el cambio social e intenta clarificar aun más la articulación del campo de la comunicación con el de la salud. Con esto, Ríos Hernández aporta al aclarar el concepto de la comunicación en la salud y su convergencia.
Posteriormente, explica que en Latinoamérica la comunicación en el campo de la salud ha tenido tres marcos o tendencias. En primer lugar, menciona una tendencia bajo la cual la comunicación se ha asumido como una herramienta de difusión de información. En este caso, no hay mayor diferenciación entre comunicar e informar. En segunda instancia, la comunicación entra a cumplir un papel de transformación o cambio social en tanto eje de organización y participación. En tercer y último lugar, se articula la comunicación en el campo de la salud con las redes de interacción humana como un componente fundamental.

A pesar de que se den estas tendencias tan diferenciadas, destaca que, a través del accionar de la comunicación en el campo de la salud, se han logrado índices altos de éxito en casos puntuales, como:

[...] la reducción del $50 \%$ de la mortalidad por cardiopatía isquémica mediante extensas actividades de comunicación en Karelia del Norte, Finlandia, y el aumento del uso de las soluciones de rehidratación oral en Honduras y otros países. Muchos países han aplicado con éxito la comunicación en salud para lograr una cobertura elevada de vacunación, controlar el cólera y alcanzar otras metas de salud pública. (Ríos, Hernández, 2011, p. 126)

Finalmente, el autor expone una serie de modelos y teorías que apalancan el accionar de la comunicación en el campo de la salud. En primera medida menciona el modelo de creencias de salud que, de acuerdo con el autor, fue creado con el fin de explicar las acciones preventivas que se toman en torno al tema de la salud. Este modelo se compone de tres elementos: 1) las percepciones individuales que se basan en la susceptibilidad y severidad de las enfermedades; 2) las percepciones individuales sobre los beneficios y las barreras para la toma de acciones preventivas; y por último 3) las claves disponibles para el individuo que lo podrían estimular y ocupar en una actividad preventiva de la salud.

Para este modelo funcionan las percepciones individuales, los factores modificantes y la 
probabilidad de acción como campos fundamentales. La crítica a este modelo, según el autor, es que se trata de un derrotero que pone gran énfasis en creencias abstractas, toda vez que basa sus consideraciones en el concepto de percepción como elemento fundamental en la ejecución de un cambio. Aun así, continúa siendo un modelo con gran prestigio a nivel mundial.

Posteriormente, menciona el modelo Precede de la década de 1980 desarrollado por Green, Kreuter, Deeds y Partridge. Este modelo es importante toda vez que permite identificar los factores que influyen en los comportamientos que conllevan problemas de salud en la población. A través de una valoración sobre estos factores, se puede identificar su grado de importancia y posible modificación para así poder actuar sobre estos. Hay tres factores que bajo este modelo contribuyen a un posible cambio de conducta: la predisposición, los factores permitidos (contexto) y los factores de refuerzo (todos aquellos que de manera positiva o negativa influyen en la conducta de los individuos).

Menciona también el modelo de las etapas de cambio. Este modelo propone acciones desde lo individual, toda vez que considera que la conducta se interviene a partir de la rutina del individuo. Las etapas de este modelo son: pre-contemplación, contemplación, determinación, acción y mantenimiento. En la etapa de pre-contemplación el individuo no tiene consciencia sobre el problema, por lo cual no considera cambiar su conducta. En la etapa de contemplación, aunque la persona logra reconocer el problema y reflexiona sobre él, no considera aún el cambio. Para llegar a la etapa de determinación puede pasar un periodo largo previo a este tránsito. En esta etapa el individuo ya considera generar un cambio en su conducta. En la etapa de acción se evidencia un cambio del individuo en su conducta en pos de resolver el problema. Finalmente, en la etapa de mantenimiento el individuo trata de mantener su conducta y evitar recaídas que lo vuelvan a poner en situación de riesgo.
Luego, el autor acota que es relevante mencionar la teoría del aprendizaje social de Bandura, la cual se estructura como punto de encuentro entre el conductismo y el cognitivismo. En esta teoría se concibe que el individuo asume un comportamiento o conducta por medio de la observación y la toma de decisiones sobre la imitación y/o la no repetición de la acción que otro haya efectuado. En este lapso, se presenta una serie de factores que hacen que el individuo tome la decisión final sobre su accionar. La teoría da gran relevancia a la expectativa en tanto aspecto clave para influenciar una conducta. Por tal razón, se dividen las expectativas en autoeficacia y de resultados. Las expectativas de autoeficacia corresponden a las capacidades individuales para lograr algo. Las expectativas de resultados se relacionan por el alcance de unos objetivos puntuales. De este modo, una persona puede esperar alcanzar algo al cambiar su conducta (expectativa de resultado), pero puede dudar de sí mismo y de sus capacidades para lograrlo (expectativas de autoeficacia).

Por último, menciona la teoría de la acción racionalizada que se centra en las creencias, actitudes, intenciones y conductas de los individuos. Esta teoría asume que todas las conductas de los individuos están asociadas a unos patrones culturales en las que basan sus estructuras de comportamiento. La teoría propone que depende de las intenciones de los individuos frente a una situación que estos asumen un compromiso mediante sus conductas. Sus intenciones dependen de: 1) la actitud del individuo frente a la conducta relacionada con el mejoramiento de su salud; 2) la carga subjetiva o percepción que tengan los individuos de lo que es realmente importante en sus vidas. En esta teoría se abordan las dimensiones de actitud, norma subjetiva, intención y conducta.

El anterior texto propone unas bases teóricas y unos modelos metodológicos para el campo de la comunicación en salud. Otras metodologías se han adoptado frente a la prevención del riesgo desde la comunicación en el contexto latinoamericano. 
El artículo titulado "El estado del arte de la comunicación de riesgos en la región de américa latina”, desarrollado en México D.F. por Ana Rosa Moreno y Frederico Peres (2011), concibe la necesidad de reconocer la gestión del riesgo desde una base: la comunicación de los factores que influyen en la vida del ser humano, partiendo del riesgo ambiental como foco principal. Por esta razón, uno de los temas que adopta para la construcción de un estado del arte a nivel latinoamericano es el de "la comunicación de riesgos como una herramienta para la salud ambiental" (Moreno y Peres, 2011).

Para Moreno y Peres la comunicación de riesgos se define como "un proceso interactivo de intercambio de información y de opiniones entre individuos, grupos e instituciones" (Moreno y Peres, 2011, p. 54), lo cual se puede articular con la teoría de la acción racionalizada que mencionaba Ríos Hernández (2011), toda vez que ambas implican un proceso interactivo de reconocimiento de la situación a través de la información y su interiorización.

Este proceso de comunicación de riesgo implica reflexiones sobre preocupaciones, reacciones y opiniones de la situación en contexto. Este proceso comunicativo comprende todo tipo de acción que se emprenda con el fin de informar a una comunidad sobre un posible riesgo en el marco de la salud u otros, como los ambientales.

De acuerdo con lo anterior, toda iniciativa de comunicación de riesgo de cualquier naturaleza tendría que ser diseñada de tal forma que asegure, de manera primordial, que los mensajes lleguen de manera efectiva a las personas que comprenden el grupo objetivo, de manera clara y por el canal que se haya asignado. Con relación a esto, Moreno presenta una serie de actores frente a las iniciativas, sus expectativas y autoridades en el caso.

Inicialmente menciona el rol del educador, el cual tiene las expectativas de explicar e informar sobre la situación de riesgo, su autoridad consiste en impartir el conocimiento necesario. En segunda instancia está el papel del facilitador, quien tiene como expectativas animar y apoyar el proceso que el educador ha iniciado. Su autoridad consiste en señalar las posibles soluciones a las problemáticas en cuestión.

Posteriormente está el rol del socio, quien tiene la expectativa de trabajar con otros y su autoridad consiste en solucionar los problemas evidenciados por los roles anteriores. Después aparece el papel del administrador, quien tiene la expectativa de decir qué es lo que se debe hacer y su autoridad se cumple bajo la acción de prevenir o mitigar el riesgo. Finalmente aparece el regulador, quien tiene como expectativa adjudicar justicia y su la autoridad es hacer cumplir las decisiones tomadas. Con esto, Moreno y Peres evidencian metodológicamente los roles que se deben clarificar en una iniciativa de riesgo.

Del documento en mención también resulta destacable que confieren una posición protagonista a la comunicación frente a la evaluación y gestión de riesgos de tipo ambiental o de salud. Como eje central, la comunicación del riesgo y la participación es el elemento vital para los procesos de gestión y prevención de riesgos. Alrededor de ella se encuentran otros factores como lo son la implementación de políticas, evaluación de políticas, identificación de peligros, evaluación de riesgos y desarrollo de políticas. Resultaría interesante una profundización sobre cada una de estas dimensiones y su función dentro de la gestión de riesgos, no obstante, la autora pasa a caracterizar y sistematizar una serie de experiencias desarrolladas en América Latina en cuanto a la comunicación en la gestión de riesgos.

Por otra parte, el texto "Comunicación y Salud" (Silva Pintos, 2001) publicado por la revista Inmediaciones de la comunicación de Uruguay, hace un análisis optimista sobre la apertura del nuevo campo de acción de esta línea de la comunicación. El texto resalta la importancia que ganó el campo de acción de la comunicación en 
la salud en la última década del siglo pasado, adoptada como un escenario de afirmación, tanto de teorías como técnicas para que la comunicación sirva como mecanismo de difusión de información, conocimientos y prácticas que contribuyan al mejoramiento de los sistemas de salud y del bienestar de las personas. Señala que en 1996 se empezaba a legitimar el campo de la comunicación en la salud como algo relevante, no solo a nivel interpersonal entre paciente y especialista, sino que también se empezó a adoptar dentro de la agenda setting de los medios de comunicación. Esta situación marcó el advenimiento de la comunicación de manera profunda en temas de salud.

La incursión de la comunicación en la salud en la esfera mediática marcaría no solamente un escenario íntimo, sino también un protagonismo en espacios de discusión política, económica y social desde los entes de influencia en masa. Aun así, se resalta la diferencia existente entre la comunicación para la salud y el campo del periodismo científico, señala que este último implica meramente difusión y análisis de la información, mientras que la comunicación en salud exige procesos estratégicos de producción y aplicación de tácticas y técnicas comunitarias dirigidas a la prevención, protección sanitaria y a los hábitos de vida saludable, además del diseño de políticas de salud y educación en estos temas a nivel global.

Otro de los aspectos relevantes que destacan en el texto tiene que ver con la transformación de la percepción de la salud como concepto, el cual abrió paso a la comunicación con elementos sustanciales para su accionar. En este sentido, la última década del siglo pasado dio apertura conceptual a la salud al ampliar su comprensión de la esfera de enfermedad/curación al de un análisis multidimensional que abarca aspectos como alimentación, vivienda, educación, nivel socioeconómico e incluso aspectos de equidad y paz. Estos aspectos han dado paso al fomento de estilos o hábitos de vida saludable como eje fundamental para la calidad de vida de las personas.
Una de las claves que menciona Silva Pintos (2001) para que la comunicación se ejerza de una manera efectiva en el campo de la salud, ha sido el reconocimiento hecho por los especialistas en salud de la importancia de la educación para la formación colectiva en hábitos de vida saludable. Esto demuestra una articulación aplicable entre la educación y el campo de la comunicación en salud, pensada para el mejoramiento de la calidad de vida de la población y el aumento de las expectativas de vida.

La efectividad de la comunicación masiva sobre el comportamiento de las personas en colectivo es otra gran clave para su articulación con el campo de la salud. De acuerdo con la autora, a la comunicación se le atribuyen propiedades efectivas para la configuración de valores, conductas y creencias en la visión del mundo y el desarrollo que las personas efectúan. Esto es algo esencial para el cambio o estructuración de hábitos de vida saludable en las comunidades. De este modo, se trata de una de las mayores razones de que exista la comunicación en el campo de la salud.

Teniendo en cuenta lo anterior, es posible analizar la comunicación para la salud en contextos sociales. El texto "Comunicación para la salud del pueblo" de Luis Ramiro Beltrán (2010) recoge la experiencia en un foro desarrollado por la Fundación Santa Fe de Bogotá, al cual fue invitado el autor y cuyo tema central fue la información y la comunicación para la seguridad social en salud, el autor evoca en uno de sus principales temas la pregunta: ¿qué es la comunicación para la salud? Beltrán afirma que la comunicación para la salud

[...] consiste de la aplicación, planificada y sistemática, de instrumentos de comunicación social al logro de comportamientos de la población compatibles con sus necesidades de bienestar y con las finalidades de servicio expresadas en políticas, estrategias y planes de salud pública. (Beltrán, 2010, p. 35)

Ofrece entonces una mirada social a la comunicación que la plantea como un mecanismo de 
intervención que busca ejercer, a gran escala, una influencia sociocultural que permita la adquisición de unos conocimientos específicos, unas actitudes y unas prácticas favorables enfocadas en la salud pública. Por otro lado, desde una mirada profesional, propone que la comunicación en el campo de la salud supone el empleo de los mecanismos y medios de comunicación masiva para encaminar los objetivos de la salud pública. De este modo, la comunicación para la salud es heredera de la antigua educación para la salud, en lo que Beltrán define como una mezcla entre arte y ciencia.

Para hablar de la comunicación y la salud en contextos educativos, uno de los documentos que de manera interesante describe ciertas aristas del tema se denomina "La comunicación para la salud como disciplina en las universidades estadounidenses" (Alcalay, 1999); si bien data de 1999, caracteriza la forma en la que el campo de la comunicación en salud penetró los escenarios universitarios en los Estados Unidos.

Según Alcalay (1999), la incursión de la comunicación en el campo de la salud dentro de las universidades estadounidenses se dio de una manera autodidacta, luego de un interés genuino sobre la profundización en esta articulación teórica y conceptual. De manera paulatina, diversos programas de formación postgradual en el campo de la comunicación y salud cobraron prestigio, como la especialización en comunicaciones para la salud de la Universidad de California en Davis y el programa de magíster en Comunicación para la salud de la Universidad de Emerson. Ambos programas académicos son descritos en el texto y destacados por su formación y el campo de acción del profesional especializado en esta área.

A manera de conclusión, Alcalay resalta que la incursión de la comunicación en el campo de la salud en los escenarios académicos de los Estados Unidos no se dio por un desarrollo planificado, sino por una necesidad que emergió del campo de forma reactiva e imperativa, para incluir los aspectos y alcances de la comunicación para la promoción de la salud en las personas. Por otro lado, las características propias del campo de la comunicación en la salud traen consigo un espacio idóneo para el desarrollo de investigaciones de corte interdisciplinario, toda vez que incluye aspectos técnicos, teóricos, metodológicos y prácticos que devienen de diferentes disciplinas.

En la misma línea de la comunicación y la salud en escenarios académicos, el artículo "Hábitos de vida saludable en la población universitaria" (Sánchez-Ojeda y De Luna-Bertos, 2015) desarrolla un estudio con el objetivo de conocer los estilos de vida de los jóvenes universitarios, a través de unas categorías establecidas y delimitadoras. Inicialmente, las autoras definen el estilo de vida como un "conjunto de pautas y hábitos comportamentales" que las personas desarrollan a través de sus actividades cotidianas. Pero no se quedan allí, comprenden que estos hábitos que se desarrollan desde la cotidianidad, en una línea extendida de tiempo, constituyen componentes potenciales o dimensiones de riesgo o seguridad, según sea su naturaleza.

Las dimensiones sobre las cuales desarrollaron la investigación fueron siete: alimentación, consumo de tabaco, alcohol, drogas, ejercicio físico, relaciones sexuales y seguridad vial. Su desarrollo investigativo se dio a través de revisiones documentales de investigaciones que relacionan los hábitos de vida saludable con la comunidad universitaria recabadas de tres bases de datos (Pubmed, Scielo y Cuiden) y publicadas entre el 2002 y el 2014. Esta búsqueda minuciosa les permitió construir un panorama de las dimensiones estipuladas y producir ciertas conclusiones. Una de las conclusiones principales fue que, a pesar de que los estudiantes universitarios aceptan y dimensionan la importancia de los hábitos de vida saludable en las personas, continúan sin llevarlos a la práctica.

Las investigadoras utilizaron tres criterios metodológicos para la inclusión de documentos en su análisis: que fueran estudios de metodología 
cualitativa y cuantitativa; que la temática fuera hábitos de vida saludable en jóvenes universitarios; y que estuvieran escritos en español y/o inglés. Las conclusiones obtenidas fueron el resultado de la depuración inicial de 569 artículos producto de investigaciones previas, analizados de manera transversal desde puntos clave como título, resúmenes y conclusiones; seleccionaron cincuenta artículos que evidenciaron conclusiones que aportaban al análisis desde las dimensiones propuestas.

Se trata de un documento recopilatorio de otras investigaciones que evidencian características analizadas desde dimensiones establecidas. Esta investigación documental resulta interesante, toda vez que sienta unas bases metodológicas de análisis, categoriza los hábitos de vida saludable y los organiza mediante unas tablas de análisis que pueden ser reestructuradas y tenidas en cuenta como base para otras investigaciones que relacionan los hábitos de vida saludable con las comunidades universitarias.

Otro documento que brinda luces sobre los hábitos de vida saludable y cómo categorizarlos es la Guía de estilos de vida saludables en el ámbito laboral (Secretaría de Salud, 2016). Es una publicación del gobierno de Honduras como respuesta a la necesidad que evidenciaron de contar con un instrumento que sirviera de guía para el tema de estilos de vida saludables -puntualmente en el ámbito laboral- en la vida cotidiana de los individuos, grupos sociales y familias. A parte, una de las intenciones del documento es el fomento de un estado de bienestar en los hondureños que pertenecen a los sectores gubernamentales y privados.

Mediante el decreto N. ${ }^{\circ}$ PCM-030-2015, el ejecutivo de Honduras prioriza la actividad física regular y la alimentación saludable para que sea aplicada de manera obligatoria en todas las entidades que se inscriben en el aparato gubernamental en sus jornadas laborales. De esta forma, el documento delimita los hábitos de vida saludables en actividad física y alimentación, pero quizá lo más relevante es que se centra en los hábitos de vida saludable para el personal colaborador, algo que resultó poco común entre los abordajes estudiados en el presente estado del arte.

A pesar de que la guía se centra en dos dimensiones para marcar el derrotero de los estilos de vida saludable, tuvo como propósito instaurar programas y proyectos que se enfocaran no solo en la actividad física y en la alimentación saludable, sino también en la salud mental, consumo de alcohol y espacios libres de tabaco. El desarrollo de la guía se plantea de tal manera que, en principio, esclarecen las motivaciones por las cuales es importante integrar la promoción de la salud en el contexto laboral. Para esto, identifican tres factores fundamentales para el desarrollo de dicha promoción de salud. El primero de ellos es el que denominan "ambiente laboral", el cual corresponde al conjunto de herramientas que facilitan el desempeño de los colaboradores en la institución a partir de su manejo adecuado; el segundo corresponde al ambiente psicosocial, el cual define todos los aspectos que pueden hacer ameno o por el contrario indeseable la vida cotidiana del colaborador en la institución; finalmente, se encuentra la dimensión de la imagen corporativa, en la cual se debe proyectar que la institución vela por el bienestar de las dimensiones de producción y salud en sus colaboradores.

Posteriormente, la guía propone un diseño del proceso de implementación a un programa de contexto laboral en condiciones saludables, el cual contiene una base ética y de valores institucionales envuelto en aspectos como el compromiso, el liderazgo y la participación de los trabajadores. Alrededor de esto, se proponen ocho pasos para dicha implementación que van desde la movilización y la evaluación, pasan por la planeación y la reevaluación, hasta llegar al mejoramiento.

Después de esto, la guía ahonda en la conceptualización y descripción de la práctica del estilo de vida que permite caracterizar los tipos de estilo de vida saludable. Se proyectan lineamientos 
para el sostenimiento de un estilo de vida saludable desde lo físico, psicológico y social. Se hace gran énfasis en el campo alimenticio, se proponen hábitos saludables de consumo de alimentos $y$ se describen enfermedades provenientes de malos hábitos alimenticios y los factores de riesgo existentes. Entre esto, se enfatiza en la obesidad y su influencia en la aparición de la diabetes, el cáncer, enfermedades cerebrovasculares, etc. Posteriormente se enfatiza en la importancia de la higiene y la vida saludable, finalmente se describe la importancia y la manera correcta de implementar la pausa activa como factor que se debe introducir para los hábitos de vida saludable del personal colaborador de las instituciones.

\section{Documentos y experiencias de comunicación y salud en Colombia}

El Documento guía alimentación saludable (Vera Rey y Hernández, 2013), en calidad de derrotero estatal -toda vez que se trata de una propuesta del Ministerio de Salud y Protección Social colombiano- presenta en cuatro capítulos una propuesta metodológica para lo que se puede llamar promoción de hábitos saludables en las personas del territorio nacional. En el primer capítulo propone lo que denominan un "análisis de situación de salud de los factores relacionados con enfermedades no transmisibles", para lo cual parten del hecho de que las enfermedades no transmisibles son una de las principales causas de muerte a nivel mundial y Colombia no es la excepción. Para esto, reconocen que la alimentación es un factor estructural susceptible a cambios, de acuerdo con las fluctuaciones sociales de los territorios.

De acuerdo con lo anterior, hacen un énfasis inicial en el factor de alimentación a nivel nacional, toman como base la Encuesta Nacional de Situación Nutricional, la cual tuvo lugar en el país en el 2010 y la Estrategia Mundial sobre Régimen Alimentario, Actividad física y Salud (Organización Mundial de la Salud [oms], 2004). En este capítulo se enfatiza en la alimentación saludable y se caracterizan temas como la situación mundial, la situación colombiana en contexto con la región, los determinantes sociales de la salud, adicionalmente se puntualiza la situación nacional.

Posteriormente, el segundo capítulo presenta lo que denominan una 'hoja de ruta' en la cual plantean estrategias para la reducción del consumo de sal y ahondan en aspectos como vivienda, educación, ámbito laboral, manejo con los individuos en la primera infancia, entre otros aspectos que complementan estos factores. En el tercer capítulo muestran indicadores sobre los lineamientos que se proponen en la guía. El último capítulo evidencia las referencias bibliográficas sobre las cuales se basaron para la construcción de la guía. Es importante destacar que este documento aporta bases metodológicas sobre lo que -a manera de antecedente- se ha hecho a nivel nacional y de orden estatal para la promoción de hábitos de vida saludable.

$\mathrm{Al}$ asumir los hábitos de vida saludable como una necesidad imperiosa para el mejoramiento de la calidad de vida de las personas y la prevención de enfermedades futuras, algunas de las dimensiones anteriormente mencionadas por el documento en cuestión se enfocan en este objetivo, toda vez que las estrategias se enmarcan en el proceder adecuado de las comunidades en su cotidianidad con relación a hábitos de vida saludables.

Por otro lado, la Facultad de Medicina de la Fundación Universitaria de las Ciencias de la Salud de Bogotá llevó a cabo la investigación que se recopila en el documento "Evaluación de estilos de vida saludable en la Facultad de Medicina de la Fundación Universitaria de Ciencias de la Salud" (Mora y Múnera, 2015) en la cual se evidencia una conceptualización sobre lo que denominan universidad saludable, la cual entienden como una institución que toma como base la promoción de la salud en su comunidad para el mejoramiento de su calidad de vida al propender por el desarrollo humano. En aspectos metodológicos, la investigación 
integró a estudiantes desde el segundo semestre de pregrado hasta estudiantes de posgrados, docentes y personal administrativo de la institución. El estudio contó con una metodología cuantitativa que respondía al diseño de una encuesta adaptada al contexto de la Pontificia Universidad Javeriana y que tuvo como base el formato de la Organización Panamericana de la Salud, con el fin de generar una línea base sobre los estilos de vida saludable de la facultad.

El instrumento se aplicó de manera virtual a través del correo electrónico en las direcciones personales e institucionales, luego de seguir el debido proceso de invitación a participar y garantizar la privacidad de las respuestas de los encuestados. Los resultados se describen y grafican, evidencian hallazgos como que el $60 \%$ de los encuestados expresó no practicar una actividad física óptima y que el $70 \%$ de estos, a pesar de expresar tener unos hábitos alimenticios adecuados, en las respuestas a detalle evidencian todo lo contrario.

La conclusión sustancial a la cual llegan los investigadores con este estudio es que a pesar de que la escala de calificación enmarca a más del $70 \%$ de la muestra encuestada en hábitos de vida saludable, la revisión detallada de los ítems del instrumento evidencia que hay hábitos de vida inadecuados en los factores de actividad física, nutrición y descanso. La intención del estudio era generar esa línea base, con la que se pretendió instaurar estrategias de intervención para minimizar los factores de riesgo.

Tabla 1. Evaluación de actividad físisca, descanso y nutrición.

\section{Dominio Resultados porcentuales}

Ítem 1.1 "nunca" realiza actividad física diaria con una intensidad moderada (caminar, bailar trotar) por lo menos durante 30 minutos

\begin{tabular}{|c|c|c|c|c|c|}
\hline \multirow{5}{*}{$\begin{array}{l}\text { Actividad } \\
\text { física } \\
(\%)\end{array}$} & Docentes & $\begin{array}{l}\text { Estudiantes de } \\
\text { pregrado }\end{array}$ & $\begin{array}{l}\text { Estudiantes de } \\
\text { posgrado }\end{array}$ & Administrativos & Total \\
\hline & 38 & 40,6 & 54 & 53 & 60,5 \\
\hline & \multicolumn{5}{|c|}{ Ítem 1.2 "nunca" realiza actividad física al menos durante 30 minutos, 5 días a la semana } \\
\hline & Docentes & $\begin{array}{c}\text { Estudiantes de } \\
\text { pregrado }\end{array}$ & $\begin{array}{c}\text { Estudiantes de } \\
\text { posgrado }\end{array}$ & Administrativos & Total \\
\hline & 62 & 56 & 84 & 60 & 43 \\
\hline \multirow{3}{*}{$\begin{array}{c}\text { Descanso } \\
(\%)\end{array}$} & \multicolumn{5}{|c|}{ Ítem 3.3 "nunca" disfruto mi tiempo libre } \\
\hline & Docentes & $\begin{array}{c}\text { Estudiantes de } \\
\text { pregrado }\end{array}$ & $\begin{array}{c}\text { Estudiantes de } \\
\text { posgrado }\end{array}$ & Administrativos & Total \\
\hline & 27,5 & 36,7 & 36 & 53 & 36,6 \\
\hline \multirow{3}{*}{$\begin{array}{c}\text { Nutrición } \\
(\%)\end{array}$} & \multicolumn{5}{|c|}{ Ítem 4.4 "nunca" me siento a gusto con mi peso } \\
\hline & Docentes & $\begin{array}{c}\text { Estudiantes de } \\
\text { pregrado }\end{array}$ & $\begin{array}{l}\text { Estudiantes de } \\
\text { posgrado }\end{array}$ & Administrativos & Total \\
\hline & 27 & 24,5 & 24 & 20 & 24,5 \\
\hline
\end{tabular}

Fuente: Мora y Múnera (2015) 
En el 2006 se publicó un documento que resumía el trabajo investigativo desarrollado tres años antes en la Universidad del Valle, denominado "Hábito de fumar en los estudiantes de primeros semestres de la Facultad de Salud: características y percepciones" (Hernández, Guevara, García y Tascón, 2006), en el cual se expresaba que, por ser el campo de la salud uno de los principales responsables en la promoción de hábitos de vida saludables desde sus profesiones y profesionales, se hizo relevante identificar las percepciones y las prácticas de tabaquismo de los estudiantes de los primeros semestres de la Facultad de Salud de la Universidad del Valle, con el fin de construir y desarrollar campañas en contra del tabaquismo dentro de la universidad.

Este documento describe una investigación de metodología cuantitativa en la cual se tomó un muestreo por conveniencia de 487 individuos, los cuales fueron seleccionados por pertenecer a los primeros semestres de la Facultad. Su información se sistematizó con el programa Epi-Info 2000 y arrojó los siguientes resultados numéricos: de la muestra, el $13 \%$ eran fumadores activos; $11 \%$ eran fumadores pasivos, $4 \%$ eran ex fumadores. En cuanto a su relación directa con el deterioro de la salud, el estudio demostró que el $22 \%$ de la muestra relacionaba el tabaquismo con la hipertensión arterial. De los fumadores, el $26 \%$ incrementaron su hábito de consumo de tabaco luego de ingresar a la universidad. De la misma muestra, el $65 \%$ aseguraron que fumaban desde antes de los 17 años y que las razones por las cuales lo hacían se deben a la angustia, depresión y nerviosismo. Finalmente, el $52 \%$ de los encuestados expresaron que sí consideran que hacían falta campañas de prevención contra el tabaquismo en jóvenes en contextos universitarios.

A manera de reflexión y conclusión, el documento resolvió dos situaciones que se perciben relevantes: la primera, que para el momento de la investigación, era evidente la carencia de campañas eficaces en cuanto al tabaquismo, lo cual generaba una vulnerabilidad para los estudiantes de primeros semestre. Y en segunda instancia, que el desconocimiento de las enfermedades que acarrea el tabaquismo continuo en las personas hace que sea una práctica frecuente en los estudiantes. Por último, uno de los hallazgos importante, fue que la cantidad de fumadores pasivos en la muestra era muy cercana a la cantidad de fumadores activos.

\section{Trabajos universitarios sobre comunicación en el campo de la salud en Cali desde el 2015 en adelante}

Por otro lado, la investigación denominada "La comunicación en la relación entre el profesional de la medicina y el paciente con cáncer" (Domínguez Arias y Ríos Álvarez, 2017) fue una investigación desarrollada en la maestría en Psicología de la Salud de la Pontificia Universidad Javeriana de Cali, la cual se enmarcó en una metodología cualitativa y con un enfoque fenomenológico, el cual buscó conocer las experiencias de un grupo de personas seleccionadas que tenían relación con la enfermedad del cáncer.

Para la recolección de la información, las investigadoras escogieron la observación no participativa y la entrevista en profundidad. En algunos casos, solicitaron permiso para grabar algunas citas de pacientes con especialistas y posteriormente, recolectaron las percepciones de los participantes en la investigación.

Del trabajo de campo desarrollado, pudieron concluir que hay una carencia con respecto a un modelo de atención en salud humanizado mediante el cual los profesionales de la salud logren adquirir habilidades comunicacionales y actitudinales frente al trato que se le da a los pacientes y sus padecimientos. Esto con el fin de armonizar el respeto, la empatía y la solidaridad ante los diversos estados de salud.

El documento titulado "El papel de las tecnologías de la información y la comunicación en 
las campañas de prevención del VIH/sIDA en entornos universitarios dirigida a la población de estudiantes entre 19 y 24 años de la Facultad de Humanidades y Ciencias Sociales de la Pontificia Universidad Javeriana de Cali" (Álvarez, 2014) corresponde a un trabajo de grado en modalidad de investigación para optar al título de Comunicación Social y Periodismo de la Pontificia Universidad Javeriana de Cali, y tuvo como objetivo la caracterización del uso de las Tic en las campañas de prevención del $\mathrm{VIH} /$ SIDA para los jóvenes universitarios entre los 19 y los 24 años de edad de la Facultad de Humanidades y Ciencias Sociales de dicha institución educativa.

Esta investigación se desarrolló con una metodología cualitativa mediante entrevista semiestructurada como técnica base para la recolección y posterior análisis de la información con soporte en luces teóricas. De este ejercicio sobresalen dos conclusiones: la primera, que el internet se ha catapultado como la plataforma comunicativa más influyente para las campañas en promoción de los temas relacionados con la salud; y en segunda instancia, la investigadora propone a través de su análisis que se deben reconsiderar los procesos educativos que se desarrollan para tratar los temas de VIH/SIDA para buscar otras alternativas que se acerquen más a las experiencias reales de la vida cotidiana de los adolescentes.

Finalmente, el artículo titulado "Las redes sociales virtuales y su influencia en los hábitos saludables de alimentación y ejercicio en los jóvenes universitarios de la ciudad de Cali" (Montoya, 2017) buscó identificar la influencia de la red social Instagram y la plataforma Youtube en los cambios de estilo de vida saludable de los jóvenes universitarios de la ciudad de Cali. Para esto, se propuso la caracterización de influenciadores locales en el ámbito de los hábitos de vida saludable, la categorización de los mensajes de dichos influenciadores y la identificación del modo de influencia que estos mensajes tenían en el cambio de los hábitos y estilos de vida de los públicos objetivos en dirección de prácticas saludables.
La metodología utilizada fue cualitativa, con técnicas como la entrevista y el diseño de una matriz de caracterización de contenidos que tuvo como fin detallar acciones para el alcance de objetivos puntuales. Esta matriz constó de doce pasos: actividades, entradas, salidas, clientes, recursos, proveedores, líderes, objetivo, alcance, documentos, parámetros y requisitos. A manera de conclusión, la matriz diseñada evidenció una compatibilidad entre lo que publican los influenciadores en sus plataformas o redes sociales y el mensaje que adoptó el público objetivo. Lo cual evidenció una efectividad en la intención del emisor y lo que asimila el receptor.

\section{Conclusiones}

Como conclusión inicial se puede determinar que el desarrollo de la comunicación para la salud como area de estudio -y sobre todo de intervenciones a nivel de ciudad y país-, ha tenido un desarrollo en Latinoamérica enfocado en el análisis de modelos teóricos centrados en las formas de generar la adopción de comportamientos saludables en las personas. A nivel de Colombia, este abordaje es aún incipiente, las únicas iniciativas se presentan en unas pocas universidades y son estudiantes de las mismas instituciones quienes las han desarrollado en sus trabajos de grado.

De igual forma, en algunos paises y entornos latinoamericanos todavía se ve la comunicación para la salud como un proceso sencillo de informar y comunicar información a través de medios masivos, se quedan en campañas con mucho ruido e inversión pero que al final tienen poco impacto. Ese escaso impacto tampoco se mide debido a que se carece también de procesos finales de evaluación de estas intervenciones.

A pesar de que algunas facultades de Comunicación o Medicina del país ya están introduciendo el tema en sus programas, este panorama hace necesario que la academia fortalezca el área de la 
comunicación para la salud al ser de vital importancia para las poblaciones y para que no quede solo en manos de los gobiernos locales, que en muchos casos hacen lo que pueden y no lo que se debe hacer.

Se evidencia que el estudio de la comunicación en el campo de la salud se aborda de manera metodológica y procedimental, pero es viable analizar estos derroteros como un mecanismo en esencia, para el cambio de los estilos de vida de los individuos.

Uno de los aspectos destacables del análisis del presente estado del arte corre por cuenta de la viabilidad de la articulación conceptual entre la comunicación y el campo de la salud, lo que devela que este no es un campo de imposibles, sino un escenario de convergencia para el cambio en los hábitos de vida saludables de las personas.

Se presenta la posibilidad de realizar un análisis de comunicación estratégica para el cambio en hábitos de vida saludables, toda vez que los procedimientos aplicados desde las experiencias recopiladas corresponden a una serie de pasos previstos para el diagnóstico y posterior acción para la prevención de enfermedades y mejoramiento de la calidad de vida de las comunidades.

Por último, se evidencia la viabilidad y necesidad de una apertura de trabajo investigativo y aplicativo que articule la comunicación y la salud, como un proceso multidimensional que se enfoque en analizar de manera sectorizada los distintos factores que comprenden los estilos y/o hábitos de vida saludables de los individuos en comunidad.

\section{Referencias}

Alcalay, R. (1999). La comunicación para la salud como disciplina en las universidades estadounidenses. Revista Panamericana de Salud Pública, 5, 192-196.
Álvarez Salazar, L. (2014). El papel de las tecnologias de la información y la comunicación en las campañas de prevención del VIH/ SIDA en entornos universitarios dirigida a la población de estudiantes entre 19 y 24 años de la Facultad de Humanidades y Ciencias Sociales de la Pontificia Universidad Javeriana de Cali (trabajo final de pregrado en Comunicación). Pontificia Universidad Javeriana. Cali, Colombia. Recuperado de http://vitela.javerianacali.edu.co/bitstream/ handle/11522/3071/Papel_tecnologias_ informacion.pdf?sequence $=1$ \&isAllowed $=\mathrm{y}$

Beltrán, L. R. (2010). Comunicación para la salud del pueblo: una revisión de conceptos básicos. Estudios sobre las Culturas Contemporáneas, XVI (31), 17-65. Recuperado de https://www. redalyc.org/articulo.oa?id=31613952002

Domínguez Arias, G. M., y Ríos Álvarez, L. (2017). La comunicación en la relación entre el profesional de la medicina y el paciente con cáncer (tesis de maestría en Psicología de la Salud). Pontificia Universidad Javeriana. Cali, Colombia. Recuperado de http:// vitela.javerianacali.edu.co/bitstream/ handle/11522/8313/Comunicaci\%c3\%b3n_ relacion_entre_profesional. pdf? sequence $=1 \&$ isAllowed $=y$

Hernández, J., Guevara, C., García, M., y Tascón, J. (2006, enero-marzo). Hábito de fumar en los estudiantes de primeros semestres de la Facultad de Salud: características y percepciones. Colombia Médica, 37(1), 31-38. Recuperado de http://www.scielo.org.co/pdf/ $\mathrm{cm} / \mathrm{v} 37 \mathrm{n} 1 / \mathrm{v} 37 \mathrm{n} 1 \mathrm{a} 05 . p d f$

Mora, S. L., y Múnera, A. F. (2015, octubre-diciembre). Evaluación de estilos de vida saludable en la Facultad de Medicina de la Fundación Universitaria de Ciencias de la Salud. Repertorio de medicina y cirugía, 24(4), 267-274. Recuperado de https://revistas.fucsalud.edu.co/ index.php/repertorio/article/view/600/638

Moreno, A. R., y Peres, F. (2011). El Estado del Arte de la Comunicación de Riesgos en la Región de América Latina. Revista de Comunicación y Salud: RCyS, 1(1), 52-68. Recuperado 
de https://dialnet.unirioja.es/servlet/ articulo?codigo $=3648880$

Montoya Martínez, S. (2017). Las redes sociales virtuales y su influencia en sus hábitos saludables de alimentación y ejercicio en los jóvenes universitarios en la ciudad de Cali (trabajo final de pregrado en Comunicación). Pontificia Universidad Javeriana. Cali, Colombia. Recuperado de http://vitela.javerianacali.edu. co/bitstream/handle/11522/10492/Redes_ sociales_virtuales.pdf?sequence $=1$ \&isAllowed $=\mathrm{y}$

Organización Mundial de la Salud [oms] (2002). Informe sobre la salud en el mundo. Reducir los riesgos y promover una vida sana. Washington, D.C.: oms.

Organización Mundial de la Salud [oms] (2004). Estrategia Mundial sobre Régimen Alimentario, Actividad física y Salud. Ginebra, Suiza: oms. Recuperado de https://www.who.int/ dietphysicalactivity/strategy/eb11344/strategy_ spanish_web.pdf

Ríos Hernández, I. (2011). Comunicación en salud: conceptos y modelos teóricos. Perspectivas de la Comunicación, 4(1), 123-140. Recuperado de http://publicacionescienciassociales.ufro.cl/ index.php/perspectivas/article/view/111/94

Sánchez-Ojeda, M. A., y De Luna-Bertos, E. (2015). Hábitos de vida saludable en la población universitaria. Nutrición Hospitalaria; 31(5), 1910-1919. Recuperado de http://dx.doi. org/10.3305/nh.2015.31.5.8608
Secretaría de Salud (2016, diciembre). Guía de estilos de vida saludables en el ámbito laboral. Tegucigalpa, Honduras: Secretaría de Salud. Recuperado de https://www. paho.org/hon/index.php?option=com docman\&view=download\&alias=382-estilosvida-saludable-actividad-fisica\&category_ slug=desarrollo-humano-sostenible-y-estilos-devida-sal\&Itemid=211

Serón, P., Muñoz, S., y Lanas, F. (2010). Nivel de actividad física medida a través del Cuestionario Internacional de Actividad Física en población chilena. Revista Médica de Chile, 138(20), 12321239. Recuperado de http://dx.doi.org/10.4067/ S0034-98872010001100004

Silva Pintos, V. (2001). Comunicación y salud. Inmediaciones de la comunicación, 3(3), 119-136. Recuperado de https:// bibliotecapromocion.msp.gob.ec/greenstone/ collect/promocin/index/assoc/HASH01e2.dir/ doc.pdf

Vera Rey, A. M., y Hernández, B. (2013). Documento guía alimentación saludable. Santiago de Cali, Colombia: Ministerio de Salud y Protección Social. 